\title{
Comparative study of long-term outcomes of accelerated and conventional collagen crosslinking for progressive keratoconus
}

\begin{abstract}
Purpose To compare the long-term outcomes of accelerated corneal collagen crosslinking (CXL) to conventional CXL for progressive keratoconus.

Patients and methods Comparative clinical study of consecutive progressive keratoconic eyes that underwent either accelerated CXL $\left(9 \mathrm{~mW} / \mathrm{cm}^{2}\right.$ ultraviolet A (UVA) light irradiance for $10 \mathrm{~min}$ ) or conventional $\mathrm{CXL}$ ( $3 \mathrm{~mW} / \mathrm{cm}^{2}$ UVA light irradiance for $30 \mathrm{~min}$ ). Eyes with minimum 12 months' follow-up were included. Post-procedure changes in keratometry readings (Flat meridian: K1; steep meridian: K2), central corneal thickness (CCT), best spectacle-corrected visual acuity (BSCVA), and manifest refraction spherical equivalent (MRSE) were analysed.
\end{abstract}

Results A total of 42 eyes were included. In all, 21 eyes had accelerated CXL

${ }^{1}$ Envision Eye Centre, Sydney, New South Wales, Australia

${ }^{2}$ Sydney Eye Hospital, The University of Sydney, Sydney, New South Wales, Australia

Correspondence: D Viswanathan, Envision Eye Centre, Level 12, 187 Macquarie Street, Sydney, NSW 2000 Australia Tel: +612 923373 33; Fax: +61292326639. E-mail: viswamdeepa@ gmail.com

Received: 31 July 2017 Accepted: 9 November 2017

Published online:

8 December 2017
(20.5 \pm 5.5 months' follow-up) and 21 eyes had conventional CXL group (20.2 \pm 5.6 months' follow-up). In the accelerated CXL group, a significant reduction in $\mathrm{K} 2(P=0.02)$, however no significant change in $\mathrm{K} 1(P=0.35)$ and CCT $(P=0.62)$ was noted. In the conventional CXL group, a significant reduction was seen in $\mathrm{K} 1(P=0.01)$ and $\mathrm{K} 2(P=0.04)$, but not in CCT $(P=0.95)$. Although both groups exhibited significant reductions in $\mathrm{K} 2$ readings, no noteworthy differences were noted between them $(P=0.36)$. Improvements in BSCVA (accelerated CXL; $P=0.22$ and conventional $\mathrm{CXL} ; \boldsymbol{P}=\mathbf{0 . 2 0}$ ) and MRSE (accelerated CXL; $P=0.97$ and conventional $C X L ; P=0.54)$ were noted, however were not significant in either group.

Conclusion Accelerated and conventional CXL appear to be effective procedures for stabilising progressive keratoconus in the long-term.
JJ Males ${ }^{1,2}$ and D Viswanathan ${ }^{1}$

Eye (2018) 32, 32-38; doi:10.1038/eye.2017.296;

published online 8 December 2017

Introduction

Keratoconus is an ectatic corneal disorder characterised by progressive thinning and conical protrusion that results in irregular astigmatism and decreased vision. ${ }^{1,2}$ Collagen crosslinking (CXL) has emerged as the standard of care for progressive keratoconus over the last decade. Several studies have evaluated the clinical efficacy of the procedure and reported favourable outcomes in terms of stable corneal curvatures and improved visual acuity. ${ }^{3-5}$

CXL involves administration of riboflavin (vitamin B2) in conjunction with ultraviolet A (UVA, $365 \mathrm{~nm}$ ) causing a photochemical process that leads to photopolymerisation of collagen molecules with resultant increased corneal biomechanical rigidity and biochemical resistance. ${ }^{6,7}$ The original CXL procedure (epithelium-off Dresden protocol) includes a $30 \mathrm{~min}$ instillation of drops $(0.1 \%$ riboflavin in $20 \%$ dextran) followed by $30 \mathrm{~min}$ of UVA illumination at $3 \mathrm{~mW} / \mathrm{cm}^{2}$ for $30 \mathrm{~min}\left(5.4 \mathrm{~J} / \mathrm{cm}^{2}\right.$ dose). ${ }^{3,4}$

The requirement for epithelial removal and the lengthy duration of procedure $(60 \mathrm{~min})$ encouraged several variations of the standard protocol, including transepithelial CXL via iontophoresis (10 min of riboflavin exposure followed by $30 \mathrm{~min}$ of UVA treatment). Experimental and clinical studies have demonstrated that iontophoresis-assisted transepithelial CXL is an effective method for riboflavin impregnation of the corneal stroma and for stabilising keratoconus progression. ${ }^{8,9}$ Comparative studies however report better results with conventional CXL. ${ }^{10}$ 
More recently, accelerated CXL procedures have been developed as an alternative technique, where higher energy settings have been used. These techniques use an irradiance of upto $30 \mathrm{~mW} / \mathrm{cm}^{2}$. This permits a shortening of the overall exposure time from $30 \mathrm{~min}$ to as little as $3-10 \mathrm{~min}$ while maintaining the total radiant exposure $\left(5.4 \mathrm{~J} / \mathrm{cm}^{2}\right) .{ }^{11-19}$ Accelerated CXL may be performed in either a pulsed or a continuous manner. Pulsing the UV light during CXL

hypothetically restarts the photodynamic reaction thus achieving an additional oxygen concentration allowing more singlet oxygen release for CXL. ${ }^{20}$ Lately, iontophoresis-assisted accelerated CXL with epithelial debridement (total treatment time of $15 \mathrm{~min}$ ) has also been described with favourable preliminary results. ${ }^{21}$

Currently, most clinical studies report that accelerated CXL has an effect equivalent to or better than conventional CXL.11-13,18,22 However, a few recent studies demonstrated a significantly greater treatment effect with conventional CXL. ${ }^{14,15}$ The biomechanical effect of CXL has been demonstrated to be oxygendependent and therefore it is possible that increasing fluence to accelerate photopolymerisation may not allow adequate time for oxygen to diffuse and participate in the reaction. ${ }^{23,24}$

A recent review article compared accelerated and conventional CXL protocols and concluded that although the safety and effectiveness of the procedures with regard to stability seem to be equivalent, the ancillary measures of efficacy, including demarcation line depth and laboratory measurements do not always confirm equivalence of both protocols. ${ }^{25}$

Most studies on accelerated CXL have reported short-term results. Very few reports exist in literature with 12 months' or greater follow-up ${ }^{14,22}$ particularly in comparison to conventional CXL. ${ }^{15,18,22}$ Hence, there is a paucity of clinical data on the longitudinal outcome of accelerated CXL. The present study aims to evaluate the long-term efficacy of accelerated CXL in comparison to conventional CXL for progressive keratoconus.

\section{Materials and methods}

This study was conducted at the Envision Eye Centre, Sydney. The study protocol was approved by the human ethics committee and adhered to the principles proposed by the declaration of Helsinki.

\section{Participants}

This was a retrospective, non-randomised comparative study of progressive keratoconic eyes that underwent accelerated CXL (21 eyes) and were compared to a historic cohort of conventional CXL-treated eyes (21 eyes) performed by the same surgeon (JJM).

The inclusion criteria for either conventional or accelerated CXL were eyes with early to moderate progressive keratoconus with a corneal thickness of at least $400 \mu \mathrm{m}$. Progressive keratoconus was defined as eyes that exhibited an increase in keratometry in the steep meridian (K2) of $1.00 \mathrm{D}$ in 1 year, deterioration in visual acuity, and the need for new contact lens fitting more than once in 2 years..$^{5}$ Exclusion criteria were advanced keratoconus with stromal scarring, corneal hydrops, herpetic keratitis, autoimmune and other systemic diseases, pregnancy, and breast feeding. Eyes with minimum 12 months' follow-up after CXL were included and all eyes underwent corneal topography using a scheimpflug camera (Pentacam HR, Oculus Inc., Wetzlar, Germany). We have not considered one eye for each patient as keratoconus is an asymmetric disease.

\section{Surgical technique}

All eyes underwent epithelium-off procedures performed by the same surgeon (JJM). Accelerated CXL was done using the Innocross system; $30 \mathrm{~min}$ riboflavin (Innocross $\mathrm{R}$, IROC Innocross AG, Zug, Switzerland) presoak and $10 \mathrm{~min}$ of $370 \mathrm{~nm}, 9 \mathrm{~mW} / \mathrm{cm}^{2}$ UVA light exposure. Similarly, conventional CXL was performed using the Innocross system; $30 \mathrm{~min}$ riboflavin (Innocross $\mathrm{R}$ ) presoak and $30 \mathrm{~min}$ of $370 \mathrm{~nm}, 3 \mathrm{~mW} / \mathrm{cm}^{2}$ UVA light exposure.

\section{Measurements}

Pre- and post-procedure changes in keratometry readings (flat meridian: K1; steep meridian: K2), central corneal thickness (CCT), best spectacle-corrected visual acuity (BSCVA), and manifest refraction spherical equivalent (MRSE) were compared between both treatment groups. Each group was subdivided into two groups based on keratometry readings, those with pre-CXL K2 greater than (advanced cases) or less than $50 \mathrm{D}$ (less-advanced cases) and results were compared.

The Pentacam HR is a high-resolution Scheimpflug camera that takes multiple slit images of the anterior segment in $<2 \mathrm{~s}$ while rotating $180^{\circ}$ around the eye. It uses a measurement wavelength of $475 \mathrm{~nm}$ (blue lightemitting diode) and 25000 measurement points are obtained. Measurements were taken with subjects in a sitting position looking at the fixation target according to the manufacturer's instructions. Only scans that the Pentacam's 'Quality specification' function determined as 'OK' were included for analysis. 
Table 1 Baseline demographics

\begin{tabular}{lccc}
\hline Parameter & Accelerated CXL & Conventional CXL & P-value \\
\hline Age (years) & $25.18 \pm 7.59$ & $24.36 \pm 6.02$ & 0.69 \\
Gender (M:F) & $15: 6$ & $12: 9$ & 0.52 \\
Follow-up (months) & $20.5 \pm 5.5$ & $20.2 \pm 5.6$ & 0.87 \\
K1 (D) & $46.88 \pm 4.13$ & $44.57 \pm 3.90$ & 0.94 \\
K2 (D) & $51.19 \pm 6.01$ & $48.50 \pm 3.32$ & 0.09 \\
CCT ( $\mu$ m) & $453.13 \pm 38.53$ & $469.07 \pm 44.78$ & 0.29 \\
BSCVA (logMAR) & $0.28 \pm 0.14$ & $0.21 \pm 0.14$ & 0.06 \\
MRSE (D) & $-4.80 \pm 5.79$ & $-5.30 \pm 4.62$ & 0.14
\end{tabular}

Abbreviations: BSCVA, best spectacle-corrected visual acuity; CCT, central corneal thickness; K1, keratometry in flat meridean; K2, keratometry in steep meridean; MRSE, manifest refraction spherical equivalent.

\section{Statistics}

Statistical analysis was performed using the Graphpad Prism 6 (GraphPad Software Inc., La Jolla, CA, USA). Measurements obtained from both devices were described as mean \pm SD. The comparison between baseline parameters between groups was performed using the unpaired $t$-test and the Fisher's exact test. The differences between pre- and post-CXL values were examined using a paired $t$-test. For all analysis, a $P$-value of $<0.05$ was considered statistically significant.

\section{Results}

A total of 42 eyes were included in this study (21 eyes after accelerated CXL vs 21 eyes after conventional CXL). Both treatment groups were followed up for comparable periods of time. The accelerated CXL group had a followup of $20.5 \pm 5.5$ months (range $12-30$ months) and the conventional CXL group was followed up for $20.2 \pm 5.6$ months (range $12-28$ months).

\section{Baseline characteristics}

A comparison of baseline demographics, including age, gender, CCT, K1, K2, MRSE, and BSCVA was performed and no significant differences were noted between both groups (Table 1). The rates of atopy were $42.9 \%$ and $57.1 \%$ in the accelerated and conventional CXL groups respectively. Baseline keratoconus cone morphology was also studied. In the accelerated CXL group, the distribution was noted to be nipple cones $(28.6 \%)$ and oval cones $(71.4 \%)$, whereas in the conventional CXL group, it was nipple cones (42.9\%) and oval cones (57.1\%).

\section{Corneal curvature change}

In the accelerated CXL group, K2 (keratometry reading at the steep meridian) reduced by mean $1.11 \pm 2.01 \mathrm{D}$
Table 2 Change in parameters at final follow-up

\begin{tabular}{lccc}
\hline Parameter & $\begin{array}{c}\text { Accelerated } \\
\text { CXL }\end{array}$ & $\begin{array}{c}\text { Conventional } \\
\text { CXL }\end{array}$ & $\begin{array}{c}\text { P- } \\
\text { value }\end{array}$ \\
\hline K1 change (D) & $0.17 \pm 1.96$ & $-0.65 \pm 1.03$ & 0.11 \\
K2 change (D) & $-1.11 \pm 2.01$ & $-0.61 \pm 1.18$ & 0.36 \\
CCT change $(\mu \mathrm{m})$ & $6.00 \pm 27.93$ & $6.87 \pm 16.52$ & 0.14 \\
BSCVA change & $-0.06 \pm 0.22$ & $-0.03 \pm 0.14$ & 0.06 \\
(logMAR) & & & \\
MRSE change $(D)$ & $-2.88 \pm 3.76$ & $-0.02 \pm 0.63$ & 0.19 \\
\hline
\end{tabular}

Abbreviations: BSCVA, best spectacle-corrected visual acuity; CCT, central corneal thickness; K1, keratometry in flat meridean; K2, keratometry in steep meridean; MRSE, manifest refraction spherical equivalent.

$(P=0.02)$, whereas in the conventional group, $\mathrm{K} 2$ reduced by mean $0.61 \pm 1.18 \mathrm{D}(P=0.04)$ at final follow-up. Although both groups exhibited statistically significant reductions in keratometry readings, no significant differences were noted between them $(P=0.36$; Table 2$)$. The trend of change in $\mathrm{K} 2$ values over time in both conventional and accelerated CXL groups is shown in Figure 1. Corneal topography showing regression in keratometry values at 12 months after accelerated CXL is shown in Figure 2.

Both accelerated and conventional CXL groups were divided into advanced (pre-CXL K2 more than $50 \mathrm{D}$ ) and less-advanced (pre-CXL K2 <50 D) subgroups. There were 8 advanced and 13 less-advanced eyes in the accelerated CXL group as compared to 6 advanced and 15 less-advanced eyes in the conventional group. No significant differences in topographic response (post-CXL K2 minus pre-CXL K2) were noted between the advanced and less-advanced subgroups of both accelerated $(P=0.50)$ and conventional $(P=0.37)$ CXL groups. Because of the small subgroup sample size, these numbers are not adequately powered to detect significant differences.

During follow-up, keratometric progression was noted in 4 of 21 accelerated CXL-treated eyes (range of change in K2 values was -0.4 to $-1.1 \mathrm{D}$ ) and in 6 of 21 conventional CXLtreated eyes (range of change in $\mathrm{K} 2$ values was -0.1 to $-1 \mathrm{D}$ ).

\section{Visual acuity change}

Improvements in BSCVA and MRSE were noted in both groups, however were not statistically significant. At final follow-up, $63.2 \%$ of accelerated CXL-treated eyes gained one or more lines of BSCVA in comparison to $47.4 \%$ of conventional CXL-treated eyes (Figure 3).

\section{Corneal thickness change}

The post-procedure change in CCT did not differ significantly between both groups $(P=0.14)$. 

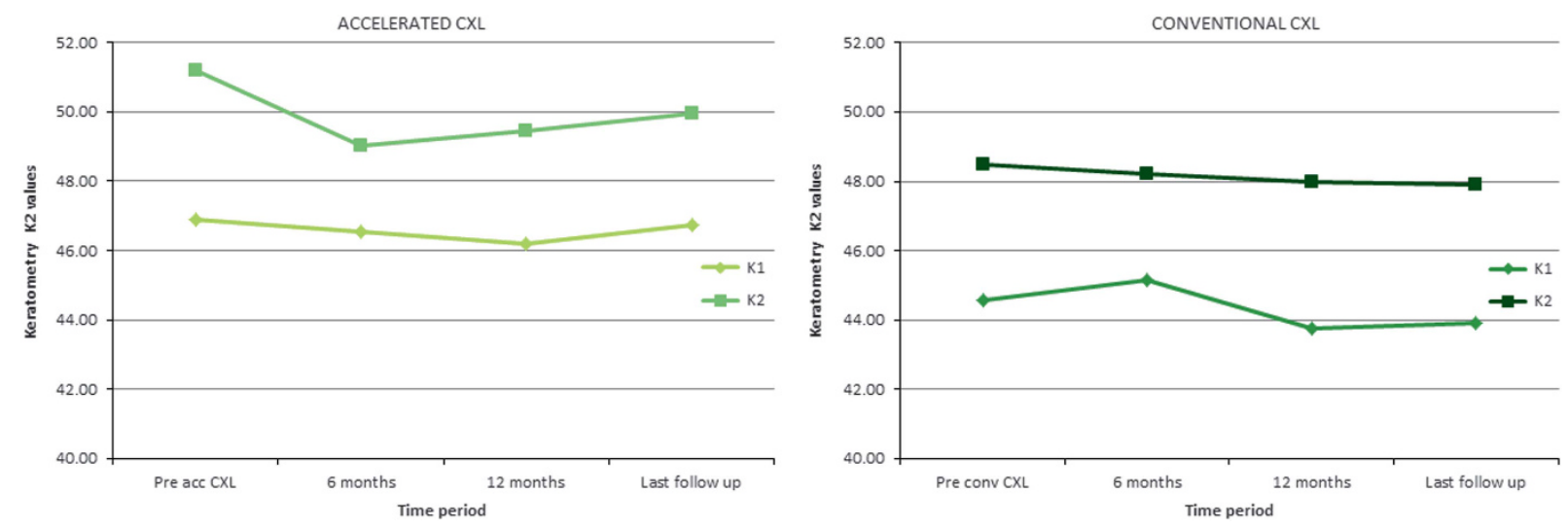

Figure 1 Trend of change in K2 values after accelerated and conventional CXL.

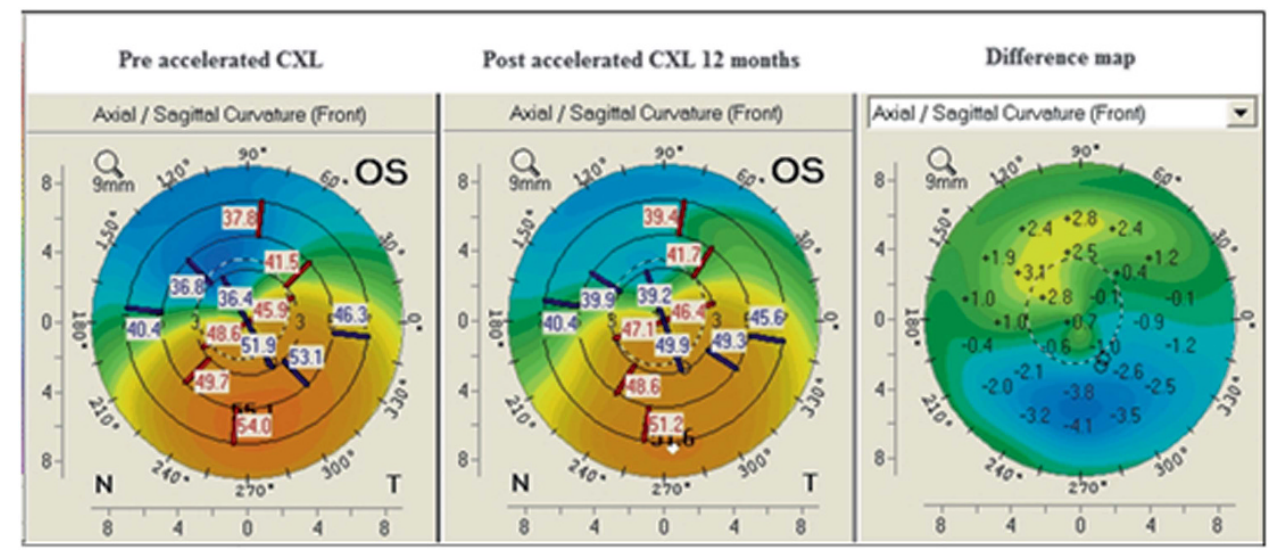

Figure 2 Corneal topography of a patient at 12 months after accelerated CXL.

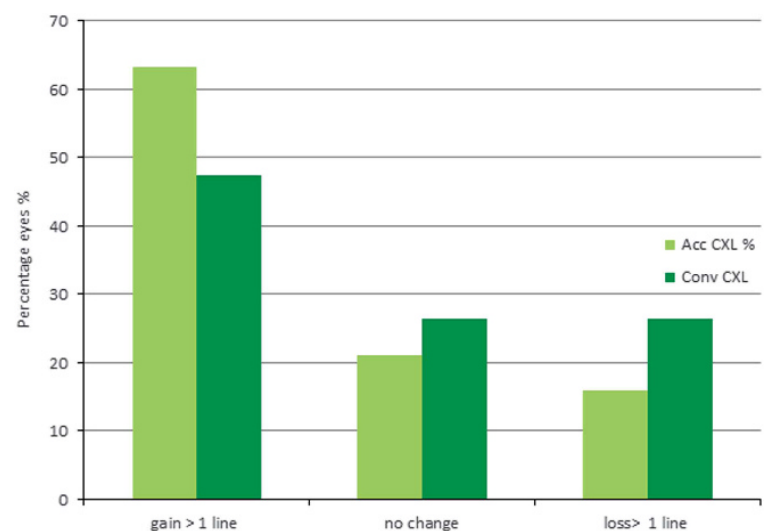

Figure 3 Comparison of change in BSCVA after accelerated and conventional CXL.

\section{Discussion}

CXL has been established as an effective procedure for progressive keratoconus by numerous studies in literature. Stability and regression of corneal curvatures and in some cases, improvement in visual acuity has been noted after conventional CXL. ${ }^{3-5}$ Accelerated CXL has been developed over the last few years with a view to reduce the overall treatment time. Laboratory studies have compared the biomechanical effects of accelerated and conventional CXL treatments on porcine corneas and found comparable effects. ${ }^{26,27}$

Recently, Hashemi et al conducted a short-term comparison between accelerated $\left(18 \mathrm{~mW} / \mathrm{cm}^{2}\right)$ and conventional methods of corneal CXL and at 6 months' follow-up, demonstrated an equivalent effect. Mean decrease in maximum keratometry values were not statistically significantly different between both groups. The mean changes in corneal biomechanical parameters, including corneal hysteresis and corneal resistance factor were also similar in both groups. ${ }^{11}$

Kanellopoulos performed a contralateral comparative study of high-fluence accelerated CXL (UVA irradiance $7 \mathrm{~mW} / \mathrm{cm}^{2}$ ) vs conventional CXL and reported a significant decrease in the steep keratometry with associated reduction in MRSE and improvement in BSCVA in both treatment groups at 6 months post treatment. $^{13}$

The long-term efficacy of accelerated CXL (UVA irradiance $9 \mathrm{~mW} / \mathrm{cm}^{2}, 10 \mathrm{~min}$ ) was assessed by Elbaz et al $^{12}$ in a cohort of 16 eyes at 6 and 12 months after the 
procedure with improvements in uncorrected visual acuity and stable keratometry values noted. Likewise, at mean follow-up of 21.7 months, improvement in keratometry and stabilisation of corrected distance visual acuity were noted after accelerated CXL $(18 \mathrm{~mW} / \mathrm{cm})$ in progressive keratoconic eyes. ${ }^{16}$

Very few studies have compared the long-term outcomes of conventional and accelerated CXL. Recently, Shetty et al ${ }^{18}$ studied the efficacy of different protocols of CXL in treating progressive keratoconus and reported comparable visual, refractive, and tomographic improvements after conventional CXL and accelerated CXL with irradiations of 9 and $18 \mathrm{~mW} / \mathrm{cm}^{2}$ at 12 months. Another study by Cummings et $a l^{22}$ noted significantly greater corneal flattening after accelerated CXL with respect to conventional CXL at 12 months. In contrast, the corneal-flattening effect of conventional CXL $(18 \mathrm{~mW} /$ $\mathrm{cm}^{2}$ ) was greater than that of accelerated CXL in a current study by Chow et al. ${ }^{15}$

The present study has a long follow-up for both accelerated (UVA irradiance $9 \mathrm{~mW} / \mathrm{cm}^{2}$ ) and conventional CXL groups, being followed up for mean 20 (range 12-30) months post treatment. Sixteen of 21 eyes $(76 \%)$ in the accelerated CXL group and 17 of 21 eyes $(81 \%)$ in the conventional CXL had follow-up $>12$ months. To the best of our knowledge, this study has the longest follow-up period for a comparative study between outcomes of both treatment groups. Statistically significant reductions in steep keratometry readings were noted after both accelerated $(51.19 \pm 6.01$ to $49.95 \pm 4.90 \mathrm{D})$ and conventional $(48.50 \pm 2.92$ to $47.89 \pm 3.62 \mathrm{D}) \mathrm{CXL}$ with no difference between both groups.

Improvements in BSCVA and MRSE were observed after both procedures, however was not statistically significant. The changes in K2 and MRSE parameters were greater with the accelerated CXL group as compared to the conventional CXL group, although not statistically significant. These changes likely contributed to greater number of patients in the accelerated CXL group gaining a line of BSCVA compared to the conventional group. In summary, the findings of this study indicate that accelerated and conventional CXL appear to be equally efficient in managing progressive keratoconus and these results are consistent with previous similar longitudinal studies. ${ }^{16,18,22}$

A current prospective, interventional study compared the functional results in two groups of patients undergoing epithelium-off pulsed accelerated CXL (plACXL) at $30 \mathrm{~mW} / \mathrm{cm}^{2}$ UVA exposure for $8 \mathrm{~min}(1 \mathrm{~s}$ on/ $1 \mathrm{~s}$ off) vs continuous light accelerated CXL (cl-ACXL) at $30 \mathrm{~mW} / \mathrm{cm} 2$ for $4 \mathrm{~min} .{ }^{28}$ Results at 12 months' follow-up demonstrated keratoconus stability in both groups. However, deeper stromal penetration and better functional outcomes were noted in the pl-ACXL group indicating that pulsed light optimised intraoperative oxygen availability thereby improving postoperative results. The topographic simulated mean $K$-value demonstrated a statistically significant reduction by -1.2 D $(P=0.049)$ after pl-ACXL, whereas an insignificant reduction by $-0.13 \mathrm{D}$ was noted after $\mathrm{cl}-\mathrm{ACXL}(P=0.088)$. In comparison, in the present study, $\mathrm{K} 2$ reduced by mean $1.11 \mathrm{D}(P=0.02)$ in the accelerated CXL group and by mean $0.61 \mathrm{D}$ in the conventional group $(P=0.04)$ at final follow-up of mean 20 months. These results are however not directly comparable as higher energy doses were used in the pl-ACXL vs cl-ACXL study $\left(7.2 \mathrm{~J} / \mathrm{cm}^{2}\right.$ instead of $5.4 \mathrm{~J} / \mathrm{cm}^{2}$ ) along with different follow-up durations.

Iontophoresis-assisted CXL is being investigated in recent times with a view to further reduce procedure time. Bikbova et al ${ }^{10}$ however reported superior results with conventional CXL (mean $K$-value reduction from $47.61 \pm 3.01$ to $45.46 \pm 2.12 \mathrm{D})$ as compared to iontophoresis-assisted transepithelial CXL (riboflavin exposure for $10 \mathrm{~min}$ followed by $30 \mathrm{~mW} / \mathrm{cm}^{2}$ UVA exposure for $30 \mathrm{~min}$; mean $\mathrm{K}$-value reduction from $46.92 \pm 3.78$ to $45.95 \pm 2.79 \mathrm{D})$ in a large cohort of 149 eyes at 24 months' follow-up. The findings of the present study are similar in nature, however the reduction in keratometry values are less pronounced possibly due to the smaller number of study subjects and shorter followup duration. Lately, Vinciguerra et $a l^{21}$ studied iontophoresis-assisted accelerated CXL with epithelial debridement (UVA irradiance $10 \mathrm{~mW}$ for $9 \mathrm{~min}$, total procedure time of $15 \mathrm{~min}$ ) in 20 eyes and reported favourable results with a mean reduction of $-1.73 \mathrm{D}$ $(P=0.3)$ of maximal keratometry value at 6 months' follow-up. However, these results need to be substantiated with further long-term results.

Findings of a recent study by Chan et al ${ }^{29}$ suggest that accelerated CXL causes greater topographic flattening and is more effective in advanced keratoconus cases compared to less-advanced cases. The present study did not find any difference in topographic response between different grades of keratoconus for either conventional or accelerated CXL treatments. However, these results were limited by a small subgroup sample size.

One limitation of the present study is that the change in corneal biomechanical parameters and endothelial count was not assessed and compared between treatment groups. One other interesting aspect might have been detailed corneal imaging to compare structural changes post accelerated and conventional CXL procedures. Toubol et al ${ }^{30}$ performed in vivo corneal confocal microscopy following both procedures and reported significant differences in corneal healing with accelerated CXL producing a greater impact than conventional CXL on the anterior cornea. One of the drawbacks of conventional CXL is the prolonged exposure time 
(30 min). Accelerated CXL, being a shorter procedure with equivalent results, could offer a benefit to the patients. A larger study with emphasis on biomechanical and structural changes following both treatment protocols is recommended to further validate the results of this study.

\section{Summary}

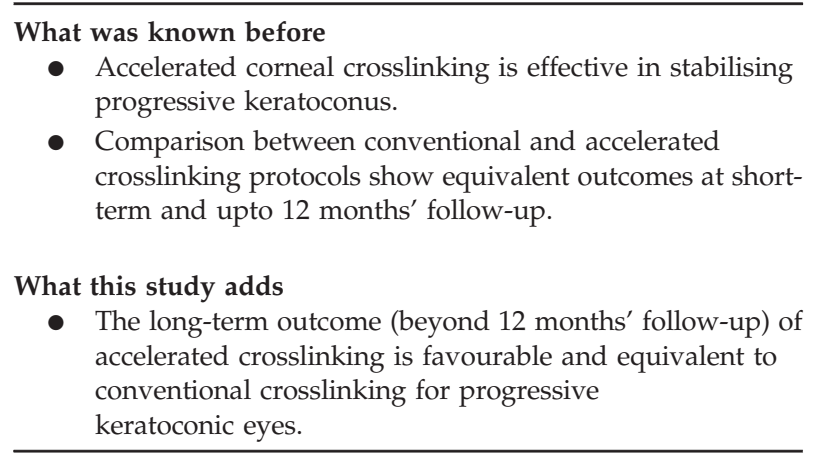

\section{Conflict of interest}

The authors declare no conflict of interest.

\section{Acknowledgements}

This study has been presented as an oral paper at the annual Royal Australian and New Zealand College of Ophthalmology (RANZCO) meeting, November 2015, Wellington, New Zealand.

\section{References}

1 Rabinowitz YS. Keratoconus. Surv Ophthalmol 1998; 42: 297-319.

2 Wollensak G, Spoerl E, Seiler T. Riboflavin/ultraviolet-ainduced collagen crosslinking for the treatment of keratoconus. Am J Ophthalmol 2003; 135: 620-627.

3 Raiskup-Wolf F, Hoyer A, Spoerl E, Pillunat LE. Collagen crosslinking with riboflavin and ultraviolet-A light in keratoconus: long-term results. J Cataract Refract Surg 2008; 34: 796-801.

4 Caporossi A, Mazzotta C, Baiocchi S, Caporossi T. Long-term results of riboflavin ultraviolet A corneal collagen cross-linking for keratoconus in Italy: the Siena eye cross study. Am J Ophthalmol 2010; 149: 585-593.

5 Viswanathan D, Males J. Prospective longitudinal study of corneal collagen crosslinking in progressive keratoconus. Clin Experiment Ophthalmol 2013; 41(6): 531-536.

6 Wollensak G, Spoerl E, Seiler T. Stress strain measurements of human and porcine corneas after riboflavineultraviolet-Ainduced cross-linking. J Cataract Refract Surg 2003; 29: 1780-1785.

7 Kohlhaas M, Spoerl E, Schilde T, Unger G, Wittig C, Pillunat LE. Biomechanical evidence of the distribution of cross-links in corneas treated with riboflavin and ultraviolet A light. J Cataract Refract Surg 2006; 32: 279-283.

8 Mastropasqua L, Nubile M, Calienno R, Mattei P, Pedrotti E, Salgari $\mathrm{N}$ et al. Corneal cross-linking: intrastromal riboflavin concentration in iontophoresis- assisted imbibition versus traditional and transepithelial techniques. Am J Ophthalmol 2014; 157: 623-630.

9 Vinciguerra P, Randleman JB, Romano V, Legrottaglie EF, Rosetta P, Camesasca FI et al. Transepithelial iontophoresis corneal collagen cross-linking for progressive keratoconus: initial clinical outcomes. J Refract Surg 2014; 30(11): 746-753.

10 Bikbova G, Bikbov M. Standard corneal collagen crosslinking versus transepithelial iontophoresis-assisted corneal crosslinking, 24 months follow-up: randomized control trial. Acta Ophthalmol 2016; 94(7): e600-e606.

11 Hashemi H, Fotouhi A, Miraftab M, Bahrmandy H, Seyedian MA, Amanzadeh $\mathrm{K}$ et al. Short-term comparison of accelerated and standard methods of corneal collagen crosslinking. J Cataract Refract Surg 2015; 41(3): 533-540.

12 Elbaz U, Shen C, Lichtinger A, Zauberman NA, Goldich Y, Chan CC et al. Accelerated $(9-\mathrm{mW} / \mathrm{cm} 2)$ corneal collagen crosslinking for keratoconus-A 1-year follow-up. Cornea 2014; 33(8): 769-773.

13 Kanellopoulos AJ. Long-term results of a prospective randomized bilateral eye comparison trial of higher fluence, shorter duration ultraviolet A radiation, and riboflavin collagen cross linking for progressive keratoconus. Clin Ophthalmol 2012; 6: 97-101.

$14 \mathrm{Ng}$ AL, Chan TC, Cheng AC. Conventional versus accelerated corneal collagen cross-linking in the treatment of keratoconus. Clin Exp Ophthalmol 2016; 44(1): 8-14.

15 Chow VW, Chan TC, Yu M, Wong VW, Jhanji V. One-year outcomes of conventional and accelerated collagen crosslinking in progressive keratoconus. Sci Rep 2015; 5: 14425.

16 Alnawaiseh M, Rosentreter A, Böhm MR, Eveslage M, Eter $\mathrm{N}$, Zumhagen L. Accelerated $\left(18 \mathrm{~mW} / \mathrm{cm}^{2}\right)$ corneal collagen cross-linking for progressive keratoconus. Cornea 2015; 34(11): 1427-1431.

17 Ozgurhan EB, Akcay BI, Kurt T, Yildirim Y, Demirok A. Accelerated corneal collagen cross-linking in thin keratoconic corneas. J Refract Surg 2015; 31(6): 386-390.

18 Shetty R, Pahuja NK, Nuijts RM, Ajani A, Jayadev C, Sharma $\mathrm{C}$ et al. Current protocols of corneal collagen crosslinking: visual, refractive, and tomographic outcomes. Am J Ophthalmol 2015; 160(2): 243-249.

19 Koç M, Uzel MM, Koban Y, Tekin K, Taşlpnar AG, Ylmazbaş P. Accelerated corneal cross-linking with a hypoosmolar riboflavin solution in keratoconic thin corneas: short-term results. Cornea 2016; 35(3): 350-354.

20 Mazzotta C, Traversi C, Caragiuli S, Rechichi M. Pulsed vs continuous light accelerated corneal collagen crosslinking: in vivo qualitative investigation by confocal microscopy and corneal OCT. Eye (Lond) 2014; 28(10): 1179-1183.

21 Vinciguerra P, Romano V, Rosetta P, Legrottaglie EF, Kubrak-Kisza M, Azzolini C et al. Iontophoresis-assisted corneal collagen cross-linking with epithelial debridement: preliminary results. Biomed Res Int 2016; 2016: 3720517.

22 Cummings AB, McQuaid R, Naughton S, Brennan E, Mrochen M. Optimizing corneal cross-linking in the treatment of keratoconus: a comparison of outcomes after standard- and high-intensity protocols. Cornea 2016; 35(6): 814-822. 
23 Richoz O, Hammer A, Tabibian D, Gatzioufas Z, Hafezi F. The biomechanical effect of corneal collagen cross-linking (CXL) with riboflavin and UV-A is oxygen dependent. Trans Vis Sci Tech 2013; 2(7): 6.

24 Mrochen M. Current status of accelerated corneal crosslinking. Indian J Ophthalmol 2013; 61(8): 428-429.

25 Kymionis GD, Kontadakis GA, Hashemi KK. Accelerated versus conventional corneal crosslinking for refractive instability: an update. Curr Opin Ophthalmol 2017; 28(4): 343-347.

26 Schumacher S, Oeftiger L, Mrochen M. Equivalence of biomechanical changes induced by rapid and standard corneal cross-linking, using riboflavin and ultraviolet radiation. Invest Ophthalmol Vis Sci 2011; 52: 9048-9052.

27 Wernli J, Schumacher S, Spoerl E, Mrochen M. The efficacy of corneal crosslinking shows a sudden decrease with very high intensity UV light and short treatment time. Invest Ophthalmol Vis Sci 2013; 54: 1176-1180.

28 Mazzotta C, Traversi C, Paradiso AL, Latronico ME, Rechichi M. Pulsed light accelerated crosslinking versus continuous light accelerated crosslinking: one-year results. J Ophthalmol 2014; 2014: 604731.

29 Chan TC, Chow VW, Jhanji V, Wong VW. Different topographic response between mild to moderate and advanced keratoconus after accelerated collagen crosslinking. Cornea 2015; 34(8): 922-927.

30 Touboul D, Efron N, Smadja D, Praud D, Malet F, Colin J. Corneal confocal microscopy following conventional, transepithelial, and accelerated corneal collagen crosslinking procedures for keratoconus. J Refract Surg 2012; 28(11): 769-776. 